\title{
Respiração de pimentão amarelo sob influência do estádio de maturação e da temperatura de armazenamento
}

\author{
Silvia Antoniali ${ }^{1}$; Paulo M Leal' ${ }^{1}$; Ana Maria de Magalhães ${ }^{1}$; Rogério T Fuziki ${ }^{1}$; Juliana Sanches ${ }^{2}$ \\ ${ }^{1}$ UNICAMP/FEAGRI, C. Postal 6011, 13083-970 Campinas-SP; E-mail: antoniali@yahoo.com; ${ }^{2}$ Instituto Agronômico, Centro APTA de \\ Engenharia e Automação, C. Postal 26, 13201-970 Jundiaí-SP; E-mail: pamleal@agr.unicamp.br
}

\section{RESUMO}

Determinou-se a taxa respiratória do pimentão amarelo 'Zarco HS' sob influência do estádio de maturação na colheita e da temperatura de armazenamento. Os pimentões foram colocados em frascos hermeticamente fechados e acondicionados em 4 BODs com diferentes temperaturas: $5 ; 10 ; 15$ e $25^{\circ} \mathrm{C}$, para os produtos colhidos no verão, e $5 ; 10 ; 15$ e $20^{\circ} \mathrm{C}$, para os colhidos no inverno. Foram utilizados cinco frascos com aproximadamente $0,5 \mathrm{~kg}$ de produto por temperatura. Foi utilizado o sistema de fluxo contínuo de ar dentro dos frascos com auxílio de um fluxômetro (flow board). As leituras do $\mathrm{CO}_{2}$ foram feitas em cromatógrafo gasoso VARIAN 3400, sendo realizadas cinco leituras por temperatura durante um período de sete dias, no verão, e 8 dias, no inverno. Após o término do experimento de inverno, a temperatura das BODs foi mantida a $10^{\circ} \mathrm{C}$ para a condução do segundo estudo. Neste, pimentões com $25 \% ; 50 \% ; 75 \%$ e $100 \%$ de coloração amarela foram armazenados para determinação da taxa respiratória durante seis dias. O delineamento experimental utilizado, em ambos os estudos, foi o inteiramente casualizado com cinco repetições. $\mathrm{O}$ amadurecimento do pimentão amarelo caracterizou-se por acréscimo na taxa respiratória, sendo que a temperatura de $5^{\circ} \mathrm{C}$ resultou em maior redução deste processo, em frutos de pimentão amarelo.

Palavras-chave: Capsicum annuum L., pós-colheita, conservação.

\section{ABSTRACT}

Respiration of yellow bell pepper 'Zarco HS' under influence of the maturation stage and of the storage temperature

The respiratory rate of the yellow bell pepper 'Zarco HS' was determined under influence of the stadium of maturation at harvest and at storage temperature. The bell peppers were placed inside tightly closed flasks and conditioned in 4 BODs with different temperatures: $5 ; 10 ; 15$ and $25^{\circ} \mathrm{C}$, for the products harvested at summer, and $5 ; 10 ; 15$ and $20^{\circ} \mathrm{C}$, for harvested at winter. Five flasks were used with approximately $0.5 \mathrm{~kg}$ of product. The system of continuous air flow was used inside of the flasks with aid of a flow board. The readings of $\mathrm{CO}_{2}$ were made in gaseous chromatograph VARIAN 3400, being accomplished five readings for each temperature during a period of seven days, in the summer, and 8 days, in the winter. After the end of the winter experiment, the temperature of BODs was maintained at $10^{\circ} \mathrm{C}$ for the conduction of the second study. In this second study, bell peppers with $25 \%$; $50 \%$; $75 \%$ and $100 \%$ of yellow coloration were stored for determination of the respiratory rate for six days. The experimental design was completely randomized, with five repetitions in both studies. The ripening of the yellow bell pepper was characterized by the increment of respiratory rate, and the temperature of $5^{\circ} \mathrm{C}$ resulted in larger reduction of this process.

Keywords: Capsicum annuum L., postharvest, conservation.

(Recebido para publicação em 07 de março de 2005; aceito em 19 de setembro de 2005)

$\mathrm{O}$ pimentão está entre as 10 hortaliças mais consumidas no mercado brasileiro. No Brasil, em 2000, a área cultivada com pimentões atingiu 13,1 mil ha. A produtividade média brasileira, no período de 1996 a 2000, aumentou em $300 \%$, passando de 10,4 para 32,6 toneladas por hectare (BlatMarchizeli et al., 2003).

Nos últimos 10 anos, no estado de São Paulo, a área cultivada com pimentão aumentou $68 \%$ enquanto que o aumento na produção foi de $116 \%$. No entanto, associado ao emprego de tecnologias que visem ao aumento da produção, é necessária a implementação de iniciativas para reduzir as perdas póscolheita. Segundo dados do Instituto de Economia Agrícola do estado de São Paulo cerca de $30 \%$ de hortifrutícolas colhido são perdidos, contabilizando uma per- da anual de US\$ 5,1 bilhões (Agrianual, 2002; Blat-Marchizeli et al., 2003).

Em países de clima tropical, é indispensável o uso da refrigeração que permite retardar os eventos fisiológicos que levam a senescência dos produtos colhidos. Para Henz (1992) o uso da refrigeração associada à embalagem favorece o aumento da vida útil de frutos de pimentão cv. Magda e em concordância com este autor, Antoniali et al. (2002) verificaram que a utilização da refrigeração retarda o amadurecimento dos frutos de pimentão amarelo, aumentando sua vida de prateleira sendo técnica eficiente para conservação pós-colheita.

Segundo Aguilera \& Chirife (1994), a alta perecibilidade de frutas e hortaliças, juntamente com a ausência de condições adequadas na colheita, transporte, embalagem e armazenagem, contri- buem para o elevado percentual de perdas pós-colheita. Barros et al. (1994), relataram que, do local de produção até o consumidor, há um grande aumento no preço do produto, e conseqüentemente, qualquer perda após a colheita resulta em acréscimo no custo da comercialização.

A velocidade com que se processa a respiração é um bom indicador do potencial de conservação de frutas e hortaliças após a colheita uma vez que altas taxas respiratórias estão, geralmente, associadas à vida útil curta no armazenamento. Em síntese, a respiração consiste na decomposição oxidativa de substâncias complexas presentes nas células, como amido, açúcares e ácidos orgânicos em moléculas simples, $\mathrm{CO}_{2} \mathrm{e}$ $\mathrm{H}_{2} \mathrm{O}$, com produção de energia. Portanto, após a colheita, o fruto tem sua vida 
independente e usa como substrato para suas funções vitais as reservas acumuladas, durante o crescimento e maturação (Kluge et al., 2002).

Este trabalho teve como objetivo determinar a taxa respiratória do pimentão amarelo, 'Zarco HS', sob influência do estádio de maturação e da temperatura de armazenamento.

\section{MATERIAL E MÉTODOS}

Foram realizados três experimentos conduzidos nos laboratórios da UNICAMP, em duas épocas distintas, janeiro (verão) e junho (inverno). Foram utilizados frutos de pimentão amarelo 'Zarco HS', colhidos em estufas da Fazenda Ituaú, localizada em Salto (SP), quando apresentavam $30 \%$ a $50 \%$ de cor amarela. Os pimentões foram colocados em frascos hermeticamente fechados e acondicionados em BODs sob diferentes temperaturas. Para o primeiro experimento, realizado no verão, as temperaturas estudadas foram $5^{\circ} \mathrm{C} ; 10^{\circ} \mathrm{C}$; $15^{\circ} \mathrm{C}$ e $25^{\circ} \mathrm{C}$, para o segundo experimento, realizado no inverno as temperaturas foram $5^{\circ} \mathrm{C} ; 10^{\circ} \mathrm{C} ; 15^{\circ} \mathrm{C}$ e $20^{\circ} \mathrm{C}$ e para o terceiro experimento, onde se fixou a temperatura em $10^{\circ} \mathrm{C}$ e variou-se o estádio de maturação; 25; 50; 75 e 100\% de coloração amarela. Foram utilizados cinco frascos em cada BOD, com aproximadamente $0,5 \mathrm{~kg}$ de produto. $\mathrm{O}$ sistema de fluxo contínuo de ar, dentro dos frascos foi feito com auxílio do fluxômetro (flow board). O 'flow board', aqui denominado fluxômetro (controlador de fluxo), é uma unidade de controle de múltiplas saídas. A estrutura foi feita de madeira, os distribuidores de tubos de PVC perfurados para 25 saídas. Cada perfuração recebeu um segmento de tubo plástico, colado com silicone gel, para funcionar como entrada ou saída (Calbo, 1989). O cálculo do fluxo de ar ambiente é função da temperatura e do calor de respiração de cada produto (Claypool \& Keefer, 1942). O fluxo de ar no fluxômetro foi regulado instalando-se um capilar em cada uma das linhas de gás ligadas aos recipientes herméticos. Os capilares instalados no fluxômetro determinavam o fluxo de ar que era levado ao produto. Para o calculo deste fluxo deve-se levar em consideração um acúmulo máximo de $0,3 \%$ de $\mathrm{CO}_{2}$ no interior do frasco com uma vazão inferior à necessária para arrastar $\mathrm{O} \mathrm{CO}_{2}$ produzido pela respiração do produto para fora do frasco. Sendo assim, a partir do fluxo desejado, foram calculados os capilares a serem utilizados. O fluxo foi calculado usando a equação:

$$
\mathrm{F}=\frac{\left(\mathrm{mLCO}_{2} / \mathrm{kg} . \mathrm{h}\right) * 100 * \mathrm{M}(\mathrm{kg})}{0,3 * 1000}
$$

Onde:

$\mathrm{F}=$ fluxo em $\mathrm{L} \mathrm{h}^{-1}$;

$\mathrm{mL} \mathrm{CO}_{2-} \mathrm{kg}^{-1} \mathrm{~h}^{-1}=$ respiração citada em literatura;

100 e $0,3=$ fatores de correção para um acúmulo máximo de $0,3 \% \mathrm{CO}_{2}$; $\mathrm{M}=$ massa do produto no frasco, em kg;

$1000=$ fator para transformação de $\mathrm{mL}$ para $\mathrm{L}$.

Nas tampas de plástico dos frascos de vidro ( $26 \times 13 \mathrm{~cm}$, volume 3,5 litros) onde o produto foi acondicionado, foram feitos três furos nos quais foram adaptadas duas tubulações (entrada e saída de fluxo) e um septo para retirada de amostras que eram levadas ao cromatógrafo, para a determinação do conteúdo de $\mathrm{CO}_{2}$.

Para identificação da melhor temperatura de acondicionamento do produto, foram utilizados cinco BODs, nas quais foram colocados cinco tubos de cobre (diâmetro de $5 \mathrm{~mm}$ e aproximadamente $3 \mathrm{~m}$ de comprimento) formando uma serpentina, para que o fluxo do ar de entrada nos frascos atingisse a temperatura de armazenagem e cinco tubos plásticos (diâmetro de $8 \mathrm{~mm}$ ) para a saída de gases dos frascos. Então, cada manômetro do fluxômetro, ajustado para o fluxo correspondente à temperatura, foi conectado a estes tubos permitindo assim uma entrada e saída do ar dos frascos herméticos proporcionando o fluxo contínuo de ar desejado.

As determinações do teor de $\mathrm{CO}_{2}$ nos frascos foram feitas em cromatógrafo gasoso VARIAN 3400, utilizando-se coluna empacotada "Chromosorb 106 $60 / 80$ " com vazão de $21,43 \mathrm{ml} / \mathrm{min}$ de gás de arraste hélio, por sete dias no verão, oito dias no inverno e seis dias no experimento de estádio de maturação.

A taxa respiratória foi determinada pelas seguintes equações que envolvem as áreas dos picos produzidos pelo cromatógrafo, o fluxo de ar utilizado em cada frasco e a massa do produto:

Cálculo da porcentagem de $\mathrm{CO}_{2}$ nos frascos:

$$
\% \mathrm{CO}_{2}=\frac{(A p-A a) \text { padrão }}{\text { Apadrão }} / 10^{4}
$$

Onde: $A p=$ área do pico da amostra; $A a$ = área do pico do ar de entrada; Apadrão = área do pico do padrão utilizado; padrão = concentração em ppm do padrão utilizado.

Cálculo de $\mathrm{CO}_{2} \mathrm{em} \mathrm{mL} \mathrm{h}^{-1}$ :

$\mathrm{CO}_{2}\left(m L \cdot h^{-1}\right)=\left(\frac{\% \mathrm{CO}_{2} * \text { fluxo }}{100}\right) * 1000$

Onde: $f l u x o=$ fluxo de ar utilizado para cada frasco com o produto, em $\mathrm{L} \mathrm{h}^{-1}$;

Cálculo da atividade respiratória, em mg CO $\mathrm{kg}^{-1} \mathrm{~h}^{-1}$ :

$$
R=\left(\frac{C O_{2}\left(m L \cdot h^{-1}\right) * 1000}{M}\right) * \rho_{\mathrm{CO}_{2}}
$$

Onde: $\mathrm{R}=$ respiração do produto em $\mathrm{mg} \mathrm{CO}, \mathrm{kg}^{-1} \mathrm{~h}^{-1} ; M=$ massa do produto, em kg; $\rho_{\mathrm{CO}_{2}}=$ densidade do $\mathrm{CO}_{2}$ na temperatura estudada $\left(\mathrm{kg} \mathrm{m}^{-3}\right)$.

O delineamento experimental utilizado foi o inteiramente casualizado com 4 tratamentos $\left(5^{\circ} \mathrm{C} ; 10^{\circ} \mathrm{C} ; 15^{\circ} \mathrm{C}\right.$ e $25^{\circ} \mathrm{C}$ no verão e $5^{\circ} \mathrm{C} ; 10^{\circ} \mathrm{C} ; 15^{\circ} \mathrm{C}$ e $20^{\circ} \mathrm{C}$ no inverno) e 5 repetições com $0,5 \mathrm{~kg}$ de produto, sendo cada frasco uma unidade experimental. Os resultados foram submetidos à análise de variância e as médias comparadas pelo teste de Tukey $(\mathrm{P} \leq 0,05)$.

Após o término do experimento do inverno, as BODs foram ajustadas para a temperatura de $10^{\circ} \mathrm{C}$, a massa de produtos em $0,5 \mathrm{~kg}$ por frasco, o fluxo nos frascos internos ajustados a partir da taxa de respiração do produto para a 
temperatura de $10^{\circ} \mathrm{C}$ e regulados pelo fluxômetro com o uso dos capilares. Os pimentões, colhidos em 4 estádios de maturação, correspondentes a 25; 50; 75 e $100 \%$ de cor amarela, foram armazenados nas BODs a $10^{\circ} \mathrm{C}$, durante seis dias. Periodicamente, foi retirada uma amostra gasosa de cada frasco através dos septos, utilizando-se uma seringa própria para cromatografia, para determinação do conteúdo de $\mathrm{CO}_{2}$ no interior do frasco, em cromatógrafo a gás. $\mathrm{O}$ delineamento experimental utilizado foi o inteiramente casualizado com 4 tratamentos $(25 \% ; 50 \% ; 75 \%$ e $100 \%$ de cor amarela) e 5 repetições sendo cada frasco uma unidade experimental $(0,5 \mathrm{~kg}$ de pimentão). Os resultados foram submetidos à análise de variância e as médias comparadas pelo teste de Tukey $(\mathrm{P} \leq 0,05)$.

\section{RESULTADOS E DISCUSSÃO}

A taxa respiratória do pimentão amarelo aumentou com a temperatura (Figura 1), conforme o explicado por Phan et al. (1975), de acordo com a Lei de Van't Hoof. O produto submetido à temperatura de $5^{\circ} \mathrm{C}$ apresentou taxa respiratória de 8 até $18 \mathrm{mg} \mathrm{CO}_{2} \mathrm{~kg}^{-1} \mathrm{~h}^{-1}$ no verão, e entre 2 e $7 \mathrm{mg} \mathrm{CO}_{2} \mathrm{~kg}^{-1} \mathrm{~h}^{-1}$ no inverno. Para as demais temperaturas de armazenagem, no verão, taxa respiratória aumentou entre 20 e $45 \mathrm{mg} \mathrm{CO} \mathrm{kg}^{-1}$ $\mathrm{h}^{-1}$, a $10^{\circ} \mathrm{C}, 30$ e $68 \mathrm{mg} \mathrm{CO} \mathrm{kg}^{-1} \mathrm{~h}^{-1}$, a $15^{\circ} \mathrm{C}$, e 110 e $504 \mathrm{mg} \mathrm{CO} \mathrm{kg}^{-1} \mathrm{~h}^{-1}$, a $25^{\circ} \mathrm{C}$. No inverno, o aumento na taxa respiratória variou de 3 a 11,3 $\mathrm{mg} \mathrm{CO}_{2}$ $\mathrm{kg}^{-1} \mathrm{~h}^{-1}$, a $10^{\circ} \mathrm{C}, 5$ a $23 \mathrm{mg} \mathrm{CO} \mathrm{kg}^{-1} \mathrm{~h}^{-1}$, a $15^{\circ} \mathrm{C}$, e 9,8 a $41,7 \mathrm{mg} \mathrm{CO}_{2} \mathrm{~kg}^{-1} \mathrm{~h}^{-1}$, a $20^{\circ} \mathrm{C}$.

A $5^{\circ} \mathrm{C}$, os valores encontrados para a taxa respiratória do pimentão amarelo no verão foram semelhantes aos encontrados por Scholz et al. (1963) citado por Hardenburg et al. (1986) enquanto que no inverno, estes valores foram inferiores aos encontrados pelos autores, à mesma temperatura. Para as demais temperaturas, no verão, estes valores se mostraram bem superiores e no inverno, próximo aos encontrados por Scholz et al. (1963) citado por Hardenburg et al. (1986), para variedades do Texas, EUA. Este diferença na taxa respiratória do produto nas duas épocas climáti-

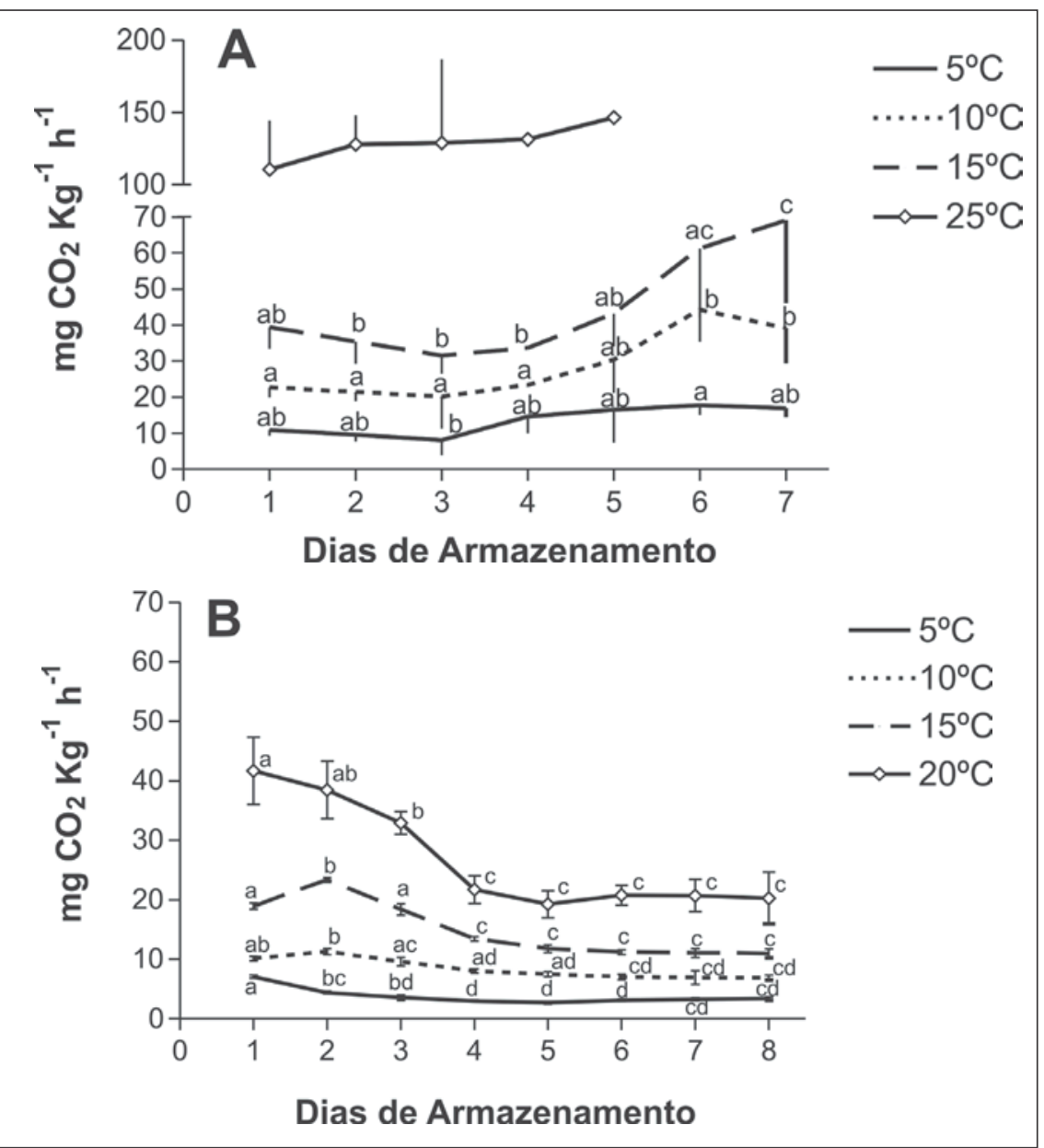

Figura 1. Taxa respiratória de pimentões amarelos 'Zarco HS' armazenados sob diferentes temperaturas, em duas épocas climáticas, verão (A) e inverno (B). Campinas, UNICAMP, 2002. Pontos seguidos de mesma letra, na mesma curva, não diferem entre si (Tukey, $\mathrm{P} \leq 0,05$ ).

cas estudadas está associada à grande diferença na temperatura durante o cultivo desta hortaliça, sendo que esta região apresenta verão quente e inverno rigoroso.

Verificou-se que em todos os tratamentos do verão, o aumento da taxa respiratória foi altamente significativo ao longo do período de armazenamento, o que confirma (Hardenburg, 1986; Kluge et al., 2002) que à medida que o fruto amadurece suas reações metabólicas aumentam, pois a respiração assume papel principal, após a colheita e é responsável pelas transformações bioquímicas que ocorrem durante o amadurecimento.

Comparando-se os dois gráficos na Figura 1, nota-se que os produtos colhidos no verão apresentaram taxa respiratória entre $8,1 \mathrm{mg} \mathrm{CO}_{2} \mathrm{~kg}^{-1} \mathrm{~h}-{ }^{1}\left(5^{\circ} \mathrm{C}\right) \mathrm{e}$ $146,5 \mathrm{mg} \mathrm{CO}_{2} \mathrm{~kg}^{-1} \mathrm{~h}^{-1}\left(25^{\circ} \mathrm{C}\right)$ enquanto para o produto colhido no inverno 2,7 mg $\mathrm{CO}_{2} \mathrm{~kg}^{-1} \mathrm{~h}^{-1}\left(5^{\circ} \mathrm{C}\right)$ e $41,7 \mathrm{mg} \mathrm{CO}_{2}$ $\mathrm{kg}^{-1} \mathrm{~h}^{-1}\left(20^{\circ} \mathrm{C}\right)$. Portanto, observa-se que o produto no verão, mesmo em baixa temperatura, apresenta uma taxa respiratória maior que o produto de inverno, aumentando seu metabolismo com o decorrer do período de armazenamento.

Para os experimentos realizados no inverno, as taxas respiratórias foram menores que as obtidas para a mesma variedade estudada no verão. Observouse, então, que o produto sofre interferência da época do ano em que for colhido, sugerindo a necessidade de uma rápida retirada do calor de campo do produto logo após a colheita principalmente para os produtos colhidos no verão.

A partir do teste de Tukey, verificouse uma diferença na taxa respiratória durante os dias de armazenamento com aumento da respiração em função dos dias de armazenamento para os produtos colhidos no verão e diminuição des- 


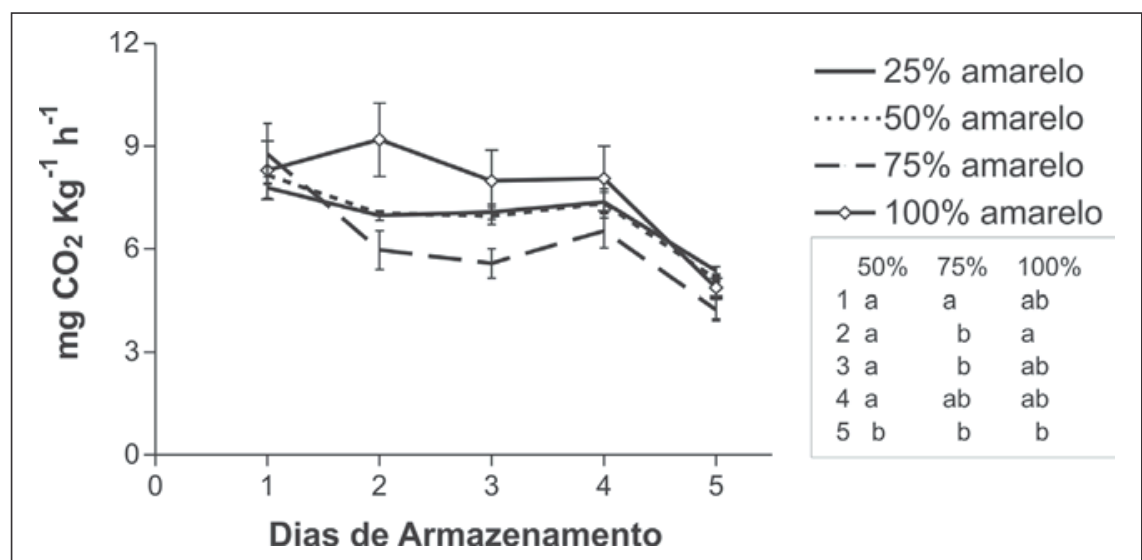

Figura 2. Taxa respiratória de pimentões amarelos 'Zarco HS' colhidos em diferentes estádios de maturação e armazenados a $10^{\circ} \mathrm{C}$. Campinas, UNICAMP, 2002.

Na tabela abaixo da legenda é mostrado o teste de Tukey $(\mathrm{P} \leq 0,05)$. Pontos seguidos de mesma letra, na mesma coluna, não diferem entre si, para os dias de armazenamento.

ta para os produtos colhidos no inverno. Quanto menor a temperatura de armazenamento do pimentão, menor foi sua taxa respiratória com diferença significativa observada através do desvio padrão (Figura 1) o que proporcionou uma maior conservação pós-colheita (Gast \& Flores, 1991).

Verificou-se que a temperatura de $5^{\circ} \mathrm{C}$ foi a mais adequada para estocagem do pimentão amarelo 'Zarco HS' colhido tanto no verão quanto no inverno. Esta resposta discorda de Hochmuch (1991), Sargent et al. (1991a), Hartz et al. (1996) e Jobling (2001) que, generalizando, afirmam que a as condições ideais de estocagem para o pimentão estão na faixa de 7 a $13^{\circ} \mathrm{C}$. Deve-se salientar que esta temperatura foi avaliada somente com base na respiração do produto, sem qualquer avaliação de custos ou efeito sobre as características de qualidade.

A taxa respiratória de pimentões amarelos em diferentes estádios de maturação variou de 4,24 a 9,19 mg CO $\mathrm{kg}^{-1} \mathrm{~h}^{-1}$, independentemente do estádio de maturação que foi colhido (Figura 2). Estes valores foram próximos aos encontrados para a mesma temperatura no experimento de inverno (3 a $11,3 \mathrm{mg}$ $\left.\mathrm{CO}_{2} \cdot \mathrm{kg}^{-1} \mathrm{~h}^{-1}\right)$, o que era esperado pois ambos os experimentos (inverno e estádio de maturação) foram realizados consecutivamente.

A respiração diminui no decorrer do armazenamento em temperaturas baixas, $10^{\circ} \mathrm{C}$, independentemente do está- dio de maturação do produto (Figura 2). Verificou-se também que a taxa respiratória apresentou diferença significativa para os tratamentos 50\%, 75\% e 100\% de cor amarela à medida que aumentava os dias de armazenamento, diminuía a taxa respiratória (Tukey, $\mathrm{P} \leq 0,05$ ). Quando se estudou a diferença entre os tratamentos nos dias de armazenagem, foi verificada uma diferença significativa para os tratamentos $100 \%$ e $75 \%$ de cor amarela, visivelmente mostrado pelo desvio padrão ( $\mathrm{Fi}$ gura 2). Para os tratamentos $25 \%$ e $50 \%$ esta diferença foi não significativa no decorrer de todo período de armazenamento e com exceção do segundo dia de armazenamento, estes dois tratamento também não diferiram dos demais.

Concluiu-se que a temperatura de $5^{\circ} \mathrm{C}$ para a estocagem do pimentão amarelo 'Zarco HS' reduziu a taxa respiratória, e conseqüentemente a atividade metabólica, aumentando sua vida útil. Esta temperatura de armazenagem para o pimentão amarelo 'Zarco HS' ainda requer estudos visando qualidade do produto e custo benefício em relação às instalações frigoríficas.

A taxa respiratória do pimentão amarelo varia em função da temperatura de armazenamento e não em relação a diferentes estádios de maturação no qual foi colhido.

\section{AGRADECIMENTOS}

À FAPESP pelo financiamento do projeto (99/04969-7), ao SAE/PIBIC pela concessão das bolsas de iniciação científica, e ao $\mathrm{CNPq}$, pela concessão da bolsa de doutorado.

\section{LITERATURA CITADA}

AGUILERA JM; CHIRIFE J. 1994. Combined methods for the preservation of foods in Latin American and the CYTED - D project. Journal of Food Engeneering, 22: 433- 444.

AGRIANUAL, Anuário da agricultura brasileira. 2002. Edição: FNP Consultoria e Comércio. São Paulo. 545 p.

ANTONIALI S; LEAL PAM; CHINCHIO G; PATARO LL; BORGHI E. 2002. Comparação de duas temperaturas de refrigeração na conservação pós-colheita de pimentão amarelo submetido a diferentes embalagens. 2002. In: FEIRA E CONGRESSO DEAR CONDICIONADO, REFRIGERAÇÃO, AQUECIMENTO E VENTILAÇÃO DO MERCOSUL. Florianópolis. CD-Rom... Florianópolis: ASBRAV.

BARROS JCSM; GOES A; MINAMI K. 1994. Condições de conservação pós-colheita de frutos de pimentão (Capsicum annuum L.). Scientia Agricola, 51: 363-368.

BLAT-MARCHIZELI SFB; YAÑEZ LDT; COSTA CP. 2003. Pimentão: Deu oídio. Revista Cultivar Hortaliças e Frutas, 21: 10-11.

CALBO AG. 1989. Adaptação de um fluxcentro para estudos de trocas gasosas e um método de aferição de capilares. Pesquisa Agropecuária Brasileira, 24: 733-739.

CLAYPOOL LL; KEEFER RM. 1942. A colorimetric method for $\mathrm{CO}_{2}$ determination. American Society for Horticultural Science, 40: 177-186.

GAST KLB; FLORES R. 1991. Precooling produce - fruits and vegetables. In: POSTHARVEST MANAGEMENT OF COMMERCIAL HORTICULTURAL CROPS. Kansas: Cooperative Extension Service.

HARDENBURG RE; WATADA AE; WANG CY. 1986. The commercial storage of fruits, vegetables and florist and nursery stocks. Washington: Department of Agriculture, Agricultural Research Service, 136 p. (Agricultural Handbook Number 66) HARTZ TK; LESTRANGE M; MAYBERRY KS; SMITH RF. 1996. Bell Pepper Productin in California, University of California, Division of Agriculture and Natural Resources Publication 7217, Vegetable Research and Information Center, Vegetable Production Series. 3 p.

HENZ GP. 1992. Conservação pós-colheita de pimentão através do uso de embalagem e refrigeração. Horticultura Brasileira, 10: 110-112.

HOCHMUTH G. 1991. Florida Greenhouse Vegetale Production Handbook, vol. 3. Florida: Cooperative Extension Service Publication SP-48. JOBLING J. 2001. Modified atmosphere packing: not as simple as it seems. Sydney. $3 \mathrm{p}$. KLUGE RA; NACHTIGAL JC; FACHINELLO JC; BILHALVA AB. 2002. Fisiologia e manejo pós-colheita de frutas de clima temperado. Campinas: Livraria e Editora Rural, 214 p.

SARGENT SA; RITENOUR MA and BRECHT JK. 1991. Handling, Cooling and Sanitation Techniques for Maintening Pstharvest Quality. Vegetables Crops Dept. University of Florida Cooperative Extension Service - Institute of Food and Agricultural Sciences, Gainesville. SSVEC47. April $1 \mathrm{p}$. 